\title{
High Thermal Amplitude Red Blood Cell Agglutinating Cold Type Autoantibodies in a Case of Severe Acute Respiratory Syndrome Coronavirus 2 Pneumonia and Multiorgan Failure
}

\author{
Hatem Hassanein ${ }^{\mathrm{a}}$, Julio Hajdenberga, b
}

\begin{abstract}
A 48-year-old man diagnosed with multiorgan failure and severe acute respiratory syndrome coronavirus 2 (SARS-CoV-2) pneumonia developed anemia and polyclonal cold agglutinins that reacted at 37 ${ }^{\circ} \mathrm{C}$. He recovered after a 2-month hospitalization where he received intensive care support. Cold agglutinins resolved after 2 weeks of supportive care. As red blood cell (RBC) cryptic antigens and warm type autoimmune hemolysis have been recently described in coronavirus disease 2019 (COVID-19) patients, we believe this mechanism may also give rise of clinically detectable cold immunoglobulin $\mathrm{M}$ (IgM) autoantibodies. Given the thermal amplitude of this particular agglutinin we believe it is possible that in vivo $\mathrm{RBC}$ agglutination could contribute to disease severity.
\end{abstract}

Keywords: Cold agglutinins; COVID-19; SARS-CoV-2; IgM; Autoantibodies; Thermal amplitude

\section{Introduction}

Several recent case reports have described severe acute respiratory syndrome coronavirus 2 (SARS-CoV-2)-related immunoglobulin $\mathrm{G}(\mathrm{IgG})$ warm type autoimmune hemolytic anemia (AIHA) [1-3]. Moreover, there is evolving evidence that SARS-CoV-2 may alter the erythrocyte surface giving rise to novel red blood cell (RBC) cryptic antigens which may play a role in these processes [4]. We recently took care of a patient that developed RBC agglutinating cold type autoantibodies.

Manuscript submitted October 13, 2020, accepted October 20, 2020

Published online November 18, 2020

aUF Health Cancer Center/Orlando Health, Orlando, FL 32806, USA ${ }^{b}$ Corresponding Author: Julio Hajdenberg, Department of Medical Oncology and Hematology, UF Health Cancer Center, 1400 S Orange Ave., Orlando, FL 32806, USA. Email: Julio.hajdenberg@orlandohealth.com

doi: https://doi.org/10.14740/jmc3608

\section{Case Report}

\section{Diagnosis and investigations}

A 48-year-old male with a clinical, radiological and microbiological diagnosis of SARS-CoV-2 infection and pneumonia required mechanical ventilation. Laboratory data initially showed anemia, significant RBC agglutination, severe anisopoikilocytosis and polychromasia (Fig. 1), and a hemoglobin concentration of $8.2 \mathrm{~g} / \mathrm{dL}$. Six days after symptom onset, the direct antiglobulin test (DAT) was positive for the polyspecific reagent to $\mathrm{C} 3 \mathrm{~b}$ and $\mathrm{C} 3 \mathrm{~d}$, but not for IgG antibodies. The cold agglutinin titer was greater than 1:2048. Thermal amplitude studies showed the cold autoantibody was reactive at $37^{\circ} \mathrm{C}$. Gross inspection of the patient's blood samples in standard ethylenediaminetetraacetic acid (EDTA) and citrate tubes showed visible agglutination (Supplementary Material 1, www.journalmc.org) which persisted when the sample was warmed above $37{ }^{\circ} \mathrm{C}$. Mycoplasma, human immunodeficiency virus (HIV), and hepatitis serologies were negative. Serum immunofixation was negative for monoclonal bands. Lactate dehydrogenase (LDH) and haptoglobin levels were normal. The antibody screen (indirect Coombs test) did not react against commercial reagent RBCs.

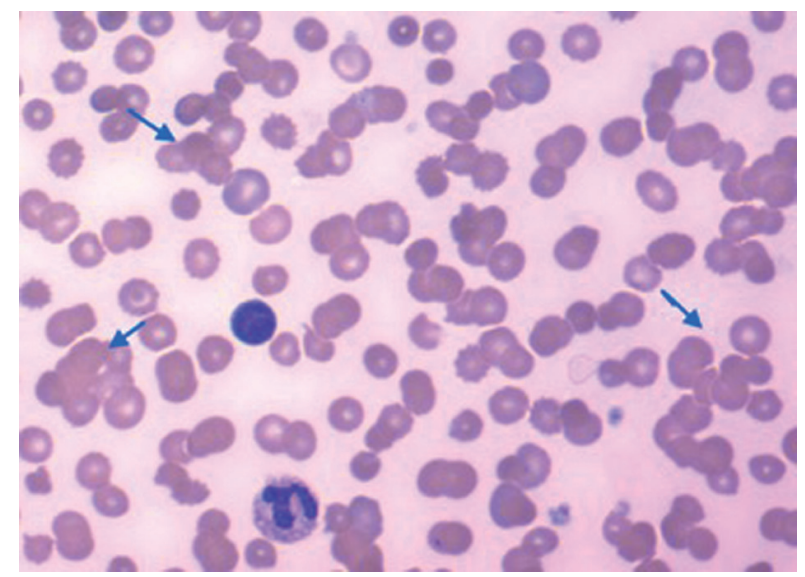

Figure 1. Peripheral blood smear showing multiple areas of red blood cell agglutination (arrows). 


\section{Treatment and outcome}

The patient remained hospitalized for approximately 8 weeks. He required transfusion of warmed blood products. After intensive care support that included mechanical ventilation, vasopressors and sedation, he gradually recovered and was discharged to a rehabilitation facility. The cold agglutinins and Coombs tests normalized 2 weeks after the initial diagnosis. Hemoglobin concentration was $9.4 \mathrm{~g} / \mathrm{dL}$ at last follow-up.

\section{Discussion}

Berzuini et al [4] recently described their experience in a Milan blood center where $46 \%$ of COVID-19 patients undergoing pre-transfusion testing were DAT-positive. Interestingly (as with our patient) these warm type antibodies did not react with standard commercial reagent RBCs (or healthy donor RBCs), but did react to RBCs prepared from DAT-negative coronavirus disease 2019 (COVID-19) patients, supporting the hypothesis that SARS-CoV-2 may alter the erythrocyte surface giving rise to novel RBC cryptic antigens.

We believe this mechanism may also give rise to clinically detectable cold (IgM) autoantibodies which may or may not result in hemolysis. In our case, there was evidence of $\mathrm{RBC}$ agglutination at physiological body temperature. Given the thermal amplitude of this particular agglutinin, it is possible that in vivo RBC agglutination contributed to the disease severity.

\section{Learning points}

As with other viral illnesses, clinically relevant IgM agglutinins may develop in patients with SARS-CoV-2 pneumonia. We observed this was a transient phenomenon that resolved as the infection ran its course.

\section{Supplementary Material}

Suppl 1. Patient's blood collected in citrate (blue top) and ethylenediaminetetraacetic acid (EDTA, purple top). Red blood cells agglutinate at room temperature.

\section{Acknowledgments}

None to declare.

\section{Financial Disclosure}

No funding was received. None of the authors have disclosures relevant to this manuscript.

\section{Conflict of Interest}

The authors declare no conflict of interest.

\section{Informed Consent}

Not applicable. The manuscript has been sufficiently de-identified to protect the patient.

\section{Author Contributions}

$\mathrm{HH}$ and $\mathrm{JH}$ jointly made the observations and conceived the idea for the article. HH wrote the initial manuscript, and recorded video material and photographed the submitted specimen. JH formatted the manuscript and edited the final version.

\section{Data Availability}

The authors declare that data supporting the findings of this study are available within the article.

\section{References}

1. Lazarian G, Quinquenel A, Bellal M, Siavellis J, Jacquy $\mathrm{C}, \operatorname{Re} \mathrm{D}$, Merabet F, et al. Autoimmune haemolytic anaemia associated with COVID-19 infection. Br J Haematol. 2020;190(1):29-31.

2. Lopez C, Kim J, Pandey A, Huang T, DeLoughery TG. Simultaneous onset of COVID-19 and autoimmune haemolytic anaemia. Br J Haematol. 2020;190(1):31-32.

3. Patel NR, Herc ES, Girgis M. Cold agglutinin disease and autoimmune hemolytic anemia with pulmonary embolism as a presentation of COVID-19 infection. Hematol Oncol Stem Cell Ther. 2020.

4. Berzuini A, Bianco C, Paccapelo C, Bertolini F, Gregato G, Cattaneo A, Erba E, et al. Red cell-bound antibodies and transfusion requirements in hospitalized patients with COVID-19. Blood. 2020;136(6):766-768. 